https://helda.helsinki.fi

\title{
Medium and long-term outcomes of gastroschisis
}

\section{Suominen, Janne}

2018-10

Suominen, J \& Rintala , R 2018 , ' Medium and long-term outcomes of gastroschisis ' ,

Seminars in pediatric surgery. , vol. 27 , no. 5 , pp. 327-329 . https://doi.org/10.1053/j.sempedsurg.2018.08.008

http://hdl.handle.net/10138/308857

https://doi.org/10.1053/j.sempedsurg.2018.08.008

publishedVersion

Downloaded from Helda, University of Helsinki institutional repository.

This is an electronic reprint of the original article.

This reprint may differ from the original in pagination and typographic detail.

Please cite the original version. 


\title{
Medium and long-term outcomes of gastroschisis
}

\author{
Janne Suominen, Risto Rintala* \\ Department of Paediatric Surgery, Children's Hospital, University of Helsinki, P.O. Box 281, Helsinki FIN-00029 HUS, Finland
}

\section{A R T I C L E I N F O}

\section{Keywords:}

Gastroschisis

Long-term

Outcomes

Follow-up

Multidisciplinary

\begin{abstract}
A B S T R A C T
As survival of gastroschisis patients has improved significantly, it has become apparent that longitudinal follow up strategies need to be developed. Problems concerning patients with gastroschisis are usually associated with gastrointestinal morbidity, but there is mounting evidence that also neurodevelopmental, cognitive, behavioral and late-onset auditory sequelae exist. The presence of associated anomalies, as well as complex features (bowel atresia, necrosis, volvulus, perforation) increase morbidity and impact long-term outcomes. Multidisciplinary follow-up is required, and the key elements of such follow-up are presented here.
\end{abstract}

(C) 2018 Elsevier Inc. All rights reserved.

\section{Introduction}

During the past few decades, outcomes in children with gastroschisis have improved significantly leading to survival rates over $90 \%$. This has raised the need for valid data for medium and longterm outcomes to guide health care professionals in planning of longitudinal follow up and in counseling of parents with a child with gastroschisis. Problems concerning patients with gastroschisis are usually associated with the gastrointestinal tract, but there is mounting evidence that neurodevelopmental, cognitive, behavioral and late-onset auditory sequelae may also exist. ${ }^{1,2}$

Complex gastroschisis, which is associated with intestinal injury in the form of atresia, volvulus, perforation, or necrosis, results in increased morbidity and a higher incidence of postoperative problems. Infectious complications, prolonged bowel obstruction, and requirement for multiple laparotomies, are common in such patients. $^{3}$ In these patients, long-term outcomes are largely determined by the extent of bowel damage at the time of the birth.

There is increasing evidence that up to one third of gastroschisis cases are associated with a variety of anomalies that may influence the prognosis significantly. ${ }^{4,5}$ Cardiac anomalies are known to be associated with omphalocele, but may be present in up to $10 \%$ of gastroschisis cases as well. Furthermore, it appears that fetuses with gastroschisis who die in utero or are terminated during pregnancy have more often associated anomalies than surviving babies with gastroschisis. ${ }^{6}$ Taken together, recent data support careful evaluation of possible associated anomalies in patients with gastroschisis, since these impact long-term outcomes significantly.

\footnotetext{
* Corresponding author.

E-mail address: risto.rintala@hus.fi (R. Rintala).
}

The bowel in gastroschisis, after prolonged exposure to amniotic fluid, is never the same as healthy newborn bowel. The bowel wall can be thickened, edematous and is often covered with fibrinous peel. Even in the absence of an associated bowel atresia, the bowel length among gastroschisis patients is shortened compared with healthy babies. Intestinal dysmotility to a variable degree is always present in newborn babies with gastroschisis.

\section{Growth of gastroschisis patients during childhood and adolescence}

Intrauterine growth is commonly retarded in patients with gastroschisis. The association between intrauterine growth retardation, bowel dysmotility, and feeding problems in gastroschisis patients, in addition to the requirement for prolonged total parenteral nutrition presents challenges for long-term growth. Median weight and length in gastroschisis patients at birth are lower compared with the non-gastroschisis population, but there is significant improvement during long-term follow-up. For the first few years, growth appears to lag behind but eventually catch-up growth improves the situation. ${ }^{7,8}$ Davies and Stringer reported normalization of weight distribution during a median 16-year follow-up and Harris et al confirmed that only $2 \%$ of the patients were underweight after a median nine years follow up. ${ }^{9,10}$ On the other hand, complex gastroschisis patients have significantly lower median body mass index in the long-term compared with simple cases. ${ }^{10}$

\section{Need for surgery in childhood and adolescence}

Surgical re-intervention is not a rare event in patients with gastroschisis, particularly in complex cases. ${ }^{11}$ Harris et al reported umbilical revision for hernia or scar repair in $30 \%$ of the patients, and bowel obstruction requiring surgical treatment in one 
fourth during a median 9-year follow up. Functional bowel obstruction and feeding intolerance are commonly seen during the first weeks after abdominal closure. In addition, acquired bowel obstruction due to adhesions is not uncommon. These adhesions can cause sub-occlusive episodes and abdominal pain leading to hospitalization. Bowel obstructions are more common during the first few months of life but can occur much later as well. ${ }^{10,12,13}$ Harris et al reported bowel obstructions early in the neonatal period whereas Risby et al reported that the majority of obstructive episodes occurred after the first year of life. van Eijck et al reviewed their 30-year experience with abdominal wall defects, reporting a $25 \%$ incidence of small bowel obstruction in patients with gastroschisis. ${ }^{12}$ In nearly all cases, adhesive bowel obstruction was confirmed at laparotomy. Multiple laparotomies, infections (sepsis, anastomotic leakage), and extensive bowel handling may increase the risk of bowel obstruction. ${ }^{12,14}$ Gore-Tex ${ }^{\circledR}$ patches used for closure in some patients do not seem to increase the risk of small bowel obstruction. ${ }^{13}$ Some complex gastroschisis babies, especially those with short bowel syndrome, will require assisted enteral feeding for a prolonged period. It may best to consider an early gastrostomy tube placement at the time of intestinal surgery in these patients, to avoid another laparotomy for gastrostomy placement. Cryptorchidism presumably develops secondary to bowel evisceration and lack of intra-abdominal pressure. While the testicle may spontaneously descend, orchidopexy is required in $10-20 \%$ of males. ${ }^{10,15}$

\section{Abdominal pain}

Chronic abdominal pain does not necessarily lead to hospitalization but is an important source of morbidity among gastroschisis patients. Questionnaire reports show that a considerable percentage of gastroschisis patients suffer from gastrointestinal symptoms. ${ }^{10,13}$ Danish gastroschisis patients had $40 \%$ hospitalization rate for gastrointestinal symptoms which is significantly more than in the general Danish pediatric population (12\%). ${ }^{13}$ Nearly half of the gastroschisis patients with median age of 9 years reported frequent abdominal pain in an Australian cohort. ${ }^{10}$ One third of gastroschisis patients reported chronic abdominal pain in the study by van Eijck et al, which is significantly higher than the healthy pediatric population. ${ }^{12}$ The knowledge on health related quality of life $(\mathrm{QoL})$ in gastroschisis patients is limited. Rankin et al. reported that children with gastroschisis had similar QoL compared with age-matched children from a background population at the age of 8-11 years, yet this study suffered from small sample size. ${ }^{16}$ Another larger study showed that simple and complicated gastroschisis patients have similar quality of life after the age of two years compared to healthy children, which is reassuring when counseling the parents. ${ }^{17}$

\section{Long-term risks of total parenteral nutrition (TPN)}

The current very low mortality rate in gastroschisis patients treated in high-resource settings renders this an inappropriate primary outcome measure. However, significant morbidity still exists and one important contributing factor is the need for prolonged TPN. Total parenteral nutrition (TPN) carries the risk for centralline associated blood stream infections (CLABSI) and is a wellrecognized cause of liver injury. ${ }^{18}$ Effective postoperative enteral feeding strategies may reduce the incidence and severity of intestinal failure-associated liver disease (IFALD) among surgical infants making TPN-related causes partly preventable. ${ }^{19}$ The inherent bowel dysmotility in newborn infants with gastroschisis often delays the initiation of enteral feeding, which may increase the risk for TPN-related problems. CLABSI was found to be a strong predictor of outcomes among 700 patients with simple gastroschisis. ${ }^{20}$
Table 1

Potential members of a multidisciplinary gastroschisis follow-up team.

\begin{tabular}{l}
\hline Pediatric surgeon \\
Pediatrician \\
Cardiologist \\
Neurologist \\
Audiologist \\
Plastic surgeon \\
Nutritionist \\
Speech therapist \\
Social worker \\
\hline
\end{tabular}

Since the main indication for a central line in gastroschisis patients is TPN, a strong emphasis needs to put on early weaning from parenteral nutrition. ${ }^{20,21}$ The achievement of enteral autonomy, which eliminates the risk of CLABSI and the hepatotoxicity of continued TPN remains a critical predictor of long-term survival in gastroschisis patients with intestinal failure.

\section{Long-term neurodevelopmental and cognitive outcomes of gastroschisis}

Research has largely focused on medical and surgical consequences of gastroschisis. Studies on neurodevelopmental and cognitive outcomes in gastroschisis patients are sparse. However, children born with gastroschisis carry known risk factors for abnormal neurodevelopmental outcomes as they are often born to young mothers, occasionally exposed to maternal vasoactive drug use (amphetamine, cocaine, aspirin, cigarettes), and born preterm and/or growth restricted. ${ }^{22}$ Medium-term outcomes of neurodevelopmental studies in gastroschisis children are encouraging, as several reports tend to show similar outcomes to those non-surgical neonatal intensive care unit patients during the first two years of life. ${ }^{7,23,24}$ Recent long-term studies propose that school-age children and adolescents with a history of gastroschisis may suffer from impaired working memory and have increased risk for behavioral problems. ${ }^{1}$ Parent-child relationships were also found to be impaired in many domains. ${ }^{1}$ Giudici et al. reported suboptimal outcomes on language developmental skills in the area of expressive language, warranting early intervention. ${ }^{25}$ Studies at pre-school age may not reveal the whole spectrum of possible neurodevelopmental disorders. A recent study from the Netherlands showed that school-aged children born with gastroschisis score significantly lower on several aspects of attention, response inhibition, executive functioning, verbal intelligence, and fine motor skills than matched controls. ${ }^{2}$ Thus, it may be beneficial to monitor neurodevelopment at early school age to improve school performance. Complex gastroschisis may lead to poorer outcomes compared with simple gastroschisis from a neurodevelopmental point of view. ${ }^{26}$ Visual impairment and sensorineural hearing loss have been reported as well, and the need for a hearing aid may be identified late on the basis of speech delay. ${ }^{15}$ It is difficult to delineate the exact etiology of the possible neurodevelopmental problems, but it can be hypothesized that nutritional challenges, repeated general anesthetics, repeated surgical interventions, and prolonged hospitalization all play a role.

\section{Long-term health care resource utilization}

Repetitive surgeries, intestinal dysfunction, feeding intolerance and infectious complications contribute to prolonged hospitalization among gastroschisis patients. Even though gastroschisis patients represent an extremely small minority of inpatients, their costs greatly exceed those of non-gastroschisis patients. ${ }^{27}$ As the natural history of complex gastroschisis is characterized by more 
Table 2

Proposed long-term follow up scheme for patients with complex gastroschisis

\begin{tabular}{|c|c|c|c|}
\hline & During neonatal hospitalization & Discharge - 1 year & $1-6$ years \\
\hline Chest x-ray, abdominal and head US & $\mathrm{x}$ (\& later as indicated) & & \\
\hline Echocardiogram & $\mathrm{x}$ (\& later as indicated) & & \\
\hline Neurodevelopmental evaluation & & & $\mathrm{x}$ (at preschool age) \\
\hline GERD Work-UP & & As indicated & \\
\hline Nutritionist & $\mathrm{x}$ & & $\mathrm{x}$ (\& later as indicated) \\
\hline Speech therapist & & & $\mathrm{x}$ \\
\hline Plastic surgeon & & & As indicated \\
\hline
\end{tabular}

US, ultrasound; GERD, gastroesophageal reflux disease;

surgical procedures and longer hospitalization, there is significant difference in total hospital charges compared with simple gastroschisis. ${ }^{28}$ Prevention of infectious complications among gastroschisis patients may represent a significant opportunity for reduction in health care costs. ${ }^{29}$

\section{Longitudinal follow-up strategies for gastroschisis patients}

As survival of gastroschisis patients has improved significantly during the past decades, it has become clear that longitudinal follow-up strategies need to be developed. In order to better counsel parents of a child with gastroschisis, risk stratifications have been introduced to categorize this disease into simple and complex categories. This has fundamental importance for the prediction of outcomes. Specific components of the multidisciplinary team around the family with a child with gastroschisis will address the long-term sequelae of this congenital anomaly (Table 1). An emphasis needs to be put on close surveillance of nutrition and proper weight gain. There is growing evidence that this anomaly may also have significant extra-intestinal sequelae, again emphasizing the need for multidisciplinary long-term follow-up. Monitoring of neurodevelopmental status of these children is advisable if there is any concern about school performance or suspicion of speech delay. Umbilical reconstruction and scar revision in some patients may be considered to relieve possible psychological stress. A proposal that includes the key elements for longterm follow-up of complex gastroschisis patients is presented in Table 2 .

\section{Conclusions}

Immediate, medium-term, and long-term survival of patients with gastroschisis are now well over 90\% in high-resource settings. However, this excellent survival should not distract clinicians from the multiple medium and long-term sequelae that can be encountered in these patients. Intestinal sequelae predominate, especially in patients born with the complex variant of the disease. However, neurodevelopmental and cognitive deficiencies may also be seen. Longitudinal multi-disciplinary follow up is therefore indicated to detect these potential abnormalities and appropriately address them.

\section{References}

1. Harris EL, Hart SJ, Minutillo C, et al. The long-term neurodevelopmental and psychological outcomes of gastroschisis: A cohort study. J Pediatr Surg. 2016:51:549-553.

2. Lap CC, Bolhuis SW, van Braeckel KN, et al. Functional outcome at school age of children born with gastroschisis. Early Hum Dev. 2017;106-107:47-52.

3. Durfee SM, Benson CB, Adams SR, et al. Postnatal outcome of fetuses with the prenatal diagnosis of gastroschisis. J Ultrasound Med. 2013;32:407412.

4. Garne E, Loane M, Dolk H, et al. Prenatal diagnosis of severe structural congenital malformations in Europe. Ultrasound Obstet Gynecol. 2005;25:6-11.
5. Benjamin B, Wilson GN. Registry analysis supports different mechanisms for gastroschisis and omphalocele within shared developmental fields. Am J Med Genet A. 2015;167A:2568-2581.

6. Akhtar J, Skarsgard ED. The Canadian pediatric surgery network (CAPSNet) associated malformations and the "hidden mortality" of gastroschisis. J Pediatr Surg. 2012;47:911-916.

7. Minutillo C, Rao SC, Pirie S, et al. Growth and developmental outcomes of infants with gastroschisis at one year of age: a retrospective study. J Pediatr Surg. 2013;48:1688-1696.

8. Lunzer H, Menardi G, Brezinka C. Long-term follow-up of children with prenatally diagnosed omphalocele and gastroschisis. J Matern Fetal Med. 2001;10:385-392.

9. Davies BW, Stringer MD. The survivors of gastroschisis. Arch Dis Child. 1997; 77:158-160.

10. Harris EL, Minutillo C, Hart S, et al. The long term physical consequences of gastroschisis. J Pediatr Surg. 2014;49:1466-1470.

11. Lap CC, Brizot ML, Pistorius LR, et al. Outcome of isolated gastroschisis; an international study, systematic review and meta-analysis. Early Hum Dev. 2016;103:209-218

12. van Eijck FC, Wijnen RM, van Goor H. The incidence and morbidity of adhesions after treatment of neonates with gastroschisis and omphalocele: a 30-year review. J Pediatr Surg. 2008;43:479-483.

13. Risby K, Husby S, Qvist N, et al. High mortality among children with gastroschisis after the neonatal period: a long-term follow-up study. J Pediatr Surg. 2017:52:431-436.

14. Choudhry MS, Grant HW. Small bowel obstruction due to adhesions following neonatal laparotomy. Pediatr Surg Int. 2006;22:729-732.

15. van Manen HendsonL, Wiley M. Early childhood outcomes of infants born with gastroschisis. I Pediatr Surg. 2013:48:1682-1687.

16. Rankin J, Glinianaia SV, Jardine J, et al. Measuring Self-reported Quality of Life in 8-11-year-old children born with gastroschisis: is the KIDSCREEN questionnaire acceptable? Birth Defects Res A Clin Mol Teratol. 2016;106:250-256.

17. Carpenter JL, Wiebe TL, Cass DL, et al. Assessing quality of life in pediatric gastroschisis patients using the pediatric quality of life inventory survey: an institutional study. J Pediatr Surg. 2016;51:726-729.

18. Willis T, Carter B, Rogers $S$, et al. High rates of mortality and morbidity occur in infants with parenteral nutrition-associated cholestasis. J Parenter Enter Nutr. 2010;34:32-37.

19. Shores DR, Alaish SM, Aucott SW, et al. Postoperative enteral nutrition guidelines reduce the risk of intestinal failure-associated liver disease in surgical infants. J Pediatr. 2018;195:140-147.

20. Youssef F, Laberge JM, Puligandla P, et al. Determinants of outcomes in patients with simple gastroschisis. J Pediatr Surg. 2017:52:710-714.

21. Fullerton BS, Hong CR, Jaksic T. Long-term outcomes of pediatric intestinal failure. Semin Pediatr Surg. 2017;26:328-335.

22. Draper ES, Rankin J, Tonks AM, et al. Recreational drug use: a major risk factor for gastroschisis? Am J Epidemiol. 2008;167:485-491.

23. South AP, Marshall DD, Bose CL, et al. Growth and neurodevelopment at 16 and 24 months of age for infants born with gastroschisis. J Perinatol. 2008:28:702-706.

24. Gorra AS, Needelman H, Azarow KS, et al. Long-term neurodevelopmental outcomes in children born with gastroschisis: the tiebreaker. J Pediatr Surg. 2012; $47: 125-129$

25. Giudici L, Bokser VS, Maricic MA, et al. Babies born with gastroschisis and followed up to the age of six years faced long-term morbidiy and impairments. Acta Paediatr. 2016;105:275-280.

26. Tosello B, Zahed M, Guimond F, et al. Neurodevelopment and healthrelated quality of life in infants born with gastroschisis: A 6-year retrospective French study. Eur J Pediatr Surg. 2017;27:352-360.

27. Hook-Dufresne DM, Yu X, Bandla V, et al. The economic burden of gastroschisis: costs of a birth defect. I Surg Res. 2015;195:16-20.

28. Arnold MA, Chang DC, Nabaweesi R, et al. Risk stratification of 4344 patients with gastroschisis into simple and complex categories. J Pediatr Surg. 2007:42:1520-1525.

29. Uribe-Leitz M, McCracken CE, Heiss KF, et al. The influence of infectious complications in gastroschisis on costs and length of stay. Am J Perinatol. 2017;34:62-69. 